\title{
The Evaluation of Vancomycin Microspheres on Intracellular Staphylococcus aureus and the Effect of Bacteria on Eukaryotic Cell Wall Permeability
}

\author{
Henry Nettey $^{1,2}$, Grace Lovia Allotey-Babington ${ }^{2}$, Martin J. D’Souza ${ }^{1}$ \\ ${ }^{1}$ Department of Pharmaceutical Sciences, Mercer University, Atlanta, USA; ${ }^{2}$ Department of Pharmaceutics and Microbiology, \\ School of Pharmacy, University of Ghana, Legon, Ghana. \\ Email: hnettey@msn.com
}

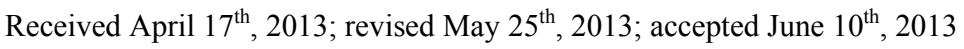

Copyright (C) 2013 Henry Nettey et al. This is an open access article distributed under the Creative Commons Attribution License, which permits unrestricted use, distribution, and reproduction in any medium, provided the original work is properly cited.

\begin{abstract}
Sepsis is a host's response to an intravascular infection; however, in most patients the disease recurs after a seemingly effective treatment. The reappearance of bacteria in the systemic circulation has been attributed to their ability to enter and hide within host endothelial cells. This study shows that internalized S. aureus is released into circulation by a possible mechanism of exocytosis through actin polymerization. Bacterial cell wall components (permeation enhancers) were significantly more effective in altering endothelial cell monolayer integrity than controls. Vancomycin has been determined to be effective in the treatment of $S$. aureus infections; however, the microencapsulated formulation of vancomycin was significantly more effective in reducing plasma and intra-tissue $S$. aureus than the conventional solution formulation. Microencapsulation of vancomycin, using albumin as a matrix, did not alter the bioactivity of the drug.
\end{abstract}

Keywords: Microencapsulation; Endothelial Cells; Permeation Enhancers; Albumin

\section{Introduction}

Death due to sepsis and peritonitis continues to be a problem in the intensive care unit. Despite aggressive approaches by modern day medicine, the mortality rate from sepsis is still very high. The pathogenesis of peritonitis and sepsis has been attributed mainly to the presence of microorganisms and the release of endotoxin into systemic circulation [1]. These invading microorganisms and their products tend to trigger extensive host defense mechanisms which include the release of cytokines and the activation of neutrophils and macrophages [2]. It has been shown that circulating microorganisms take refuge within professional as well as non-professional phagocytic cells $[3,4]$. These intracellular bacteria multiply and eventually find their way out of the cells and re-infect other cells. The mechanism by which intracellular bacteria exit host cells is still unclear, however, it has been hypothesized that the presence of bacteria and the cellular components in systemic circulation compromises the integrity of the endothelium [5]. This eventually leads to vascular leakage, multiple organ failure and death. It is the goal of this research to show that intra-endothelial
S. aureus are released by exocytosis and that actin polymerization is a possible mechanism by which this occurs.

Vancomycin, a tricyclic antibiotic, has been a key player in the antimicrobial war and is often used as the antibiotic of last resort in treating most resistant $S$. aureus infections. Vancomycin is highly water soluble and therefore lends itself to highly efficient encapsulation by albumin. Vancomycin has been combined with microspheres of tumor necrosis factor- $\alpha$ (TNF- $\alpha$ ) to effectively protect rats from $S$. aureus induced peritonitis [6]. Biodegradable polymers have been widely used as matrices for encapsulation of drugs. Albumin is a natural polymer that is biocompatible, biodegradable, and relatively non-toxic. It is water soluble and has effectively been used to encapsulate water soluble drugs [7-9]. Previous in vitro studies in our lab have shown that encapsulated vancomycin is more effective than the drug solution in killing intracellular S. aureus [10]. It has been shown that albumin microspheres are effectively phagocytosed by human macrophages [11]. The encapsulation of vancomycin would hopefully confer a sustained as well as targeted delivery of the drug, hence improving 
the therapeutic efficacy of the drug.

The goal in the treatment of peritonitis and sepsis should be an early eradication of the causative agent and a prolonged presence of the drug (within the therapeutic range) to prevent recurrence of the disease. In this study, the uptake and exocytosis of $S$. aureus by endothelial cells was evaluated. Also the effect of bacterial cell wall components on endothelial integrity was evaluated. Finally, the effect of encapsulated vancomycin in a peritonitis rat model was assessed.

\section{Materials and Methods}

Human microvascular endothelial cells (HMEC's) and culture media (MCDB131, supplemented EBM media) were obtained from the Centers for Disease Control (CDC). Lyophilized powder of S. aureus strain ATCC6538 was purchased from ATCC, Bethesda, Maryland. Tripticase soy agar, tripticase soy broth, culture flasks, petri dishes, transwell inserts (Costar \#4750), 24-well tissue culture plates, and pipettes were purchased from Fisher Scientific Company, Norcross, Georgia. Antibiotics and other drug powders were purchased from Sigma Chemical Company (St. Louis, MO). Male Sprague Dawley rats were purchased from Harlan (Indianapolis, $\mathrm{IN})$.

\subsection{Preparation of Microspheres}

In a previous study, vancomycin loaded albumin microspheres were prepared by the spray-dry method. Drug loading in the microspheres was approximately $18.6 \%$ with an encapsulation efficiency of $89 \%$ [12]. The mean microsphere size was $5 \pm 1.6 \mu \mathrm{m}$. The microspheres were used in all the experiments described below.

\subsection{Exocytosis of Intracellular S. aureus from HMEC's}

HMEC's were grown to confluency in 24-well plates and exposed to $S$. aureus in the ratio of $100: 1 / S$. aureus: HMEC's. After 1 hour of exposure, cells were washed twice with sterile phosphate buffered saline (PBS) and adhered extracellular bacteria were killed using gentamicin solution $(100 \mu \mathrm{g} / \mathrm{ml})$ for 1 hour. In a preliminary study, the infected cells were incubated at $37^{\circ} \mathrm{C}$ and at various time intervals serial dilutions of the extracellular media were plated to determine the number of exocytosed bacteria, if any [10]. Results obtained from this study were used to determine the incubation time of infected cells prior to treatment with cytochalasin D. Cytochalasin $\mathrm{D}$, a potent inhibitor of actin polymerization, was used to determine whether actin polymerization was involved in the release of bacteria from cells and hence in exocytosis.
After the gentamicin wash, HMEC's were incubated in fresh media for three hours after which they were divided into two groups. One group was treated with $1 \mu \mathrm{g} / \mathrm{ml}$ of cytochalasin D (in MCDB131, supplemented EBM media) for two hours while the other group had no cytochalasin D treatment. The cells were washed twice with PBS and incubated in HMEC media (EBM) until ready for analysis. At various time intervals, serial dilutions of the media were plated on agar and bacterial cell count determined. HMEC's were washed twice with PBS, and adhered extracellular bacteria were killed using gentamicin solution. The cells were then lysed for 1 hour with $1 \%$ triton-X 100 solution. Serial dilutions of cell lysates were also plated on agar to determine the intracellular bacterial count at each time point.

\subsection{Effect of S. aureus and Its Cell Wall Products on HMEC Permeability}

For this group of experiments HMEC's were grown to confluency on transwell inserts (Costar \#4750). The inserts were pre-treated with $20 \mu 1$ collagen, and allowed to dry in a sterile hood for two hours. $2 \times 10^{5}$ cells/insert $(0.15 \mathrm{ml})$ were plated for 72 hours, which was the time required for the cells to reach confluency. $600 \mu 1$ of media was added to each of the bottom wells. At the end of the incubation period, the media was removed from both the inserts and the bottom wells, cells were washed with PBS, and sterile Hanks Balanced Salt Solution (1\% HBSS) was added to both the inserts and the wells. The cells which had to be challenged with $S$. aureus were exposed to the bacteria for one hour (to allow uptake) after which they were washed off with HBSS. For each plate, the cells were divided into five groups, and each group was treated with $100 \mu \mathrm{l}$ of one of the following cell wall components or bacteria: Control (1\% HBSS); Lipoteichoic acid (LTA) $100 \mu \mathrm{g} / \mathrm{ml}$; Peptidoglycan (PepG) $100 \mu \mathrm{g} / \mathrm{ml} ;$ LTA + Pep G $(100 \mu \mathrm{g} / \mathrm{ml})$; and $S$. aureus (SA). The permeation of Fluorescein Isothiocynate (FITC), FITC-Dextran, FITC-Human serum albumin (FITC-HSA), and SA through the HMEC monolayer was evaluated. Fluorescein compounds of different molecular weights were used to determine the extent by which the endothelial cell monolayer is compromised. Cells in each plate were exposed to one type of fluorescent molecule or bacteria. At various time points, fluorescent intensity in the bottom wells was determined with a Cytofluorometer with the emission and excitation wavelengths set to 485 and $530 \mathrm{~nm}$ respectively. For the permeation of S. aureus, serial dilutions of the bottom wells were plated at various time intervals. The wells were washed and fresh media added. The cumulative permeation of bacteria was determined at each time point. 


\subsection{Efficacy of Vancomycin Microspheres and Solution in Rats}

The efficacy of vancomycin microspheres as compared to solution was determined in septic shock rat models. Three scenarios were evaluated: Prophylactic treatment, simultaneous treatment, and delayed treatment. In each study, the rats were divided into three groups: 1) Control group: In which rats $(\mathrm{n}=2$, one rat died before samples could be taken) were injected with blank albumin microspheres; 2) Vancomycin solution group: In which rats (n =3) were treated with vancomycin solution; 3) Vancomycin microsphere group: In which rats $(n=3)$ were treated with encapsulated vancomycin.

\subsection{Prophylactic Vancomycin Treatment}

In the prophylactic treatment study, rats were injected intraperitonially (IP), with drug solution, microspheres, or blank $(15 \mathrm{mg} / \mathrm{kg})$. Four hours after treatment, the rats were anesthetized; IP, with ketamine $(100 \mathrm{mg} / \mathrm{kg})$ and xylazine $(10 \mathrm{mg} / \mathrm{kg})$ and tail vein blood samples $(400 \mu \mathrm{l})$ were obtained. The rats were then injected, IP, with 0.5 $\mathrm{ml}$ of a suspension of $S$. aureus $\left(1.0 \times 10^{8} \mathrm{cfu} / \mathrm{ml}\right)$. Tail vein blood samples were obtained at 4, 24, 48, 72, and 96 hrs post infection. All the rats received their respective treatments of twice a day for three days. Serial dilutions of blood samples were plated on agar, and bacteremia count was obtained 24 hours after incubation at $37^{\circ} \mathrm{C}$. The rats were euthanized on day five and the liver, spleen, and lungs were excised. Each organ was immediately placed in a $1 \%$ Hanks Balanced Salt solution (HBSS) with $100 \mu \mathrm{g} / \mathrm{ml}$ of gentamicin for 30 minutes.

The organs were washed twice with HBSS, weighed, and homogenized in $10 \mathrm{ml}$ of $1 \%$ Triton-X 100 solution using a hand-held homogenizer. The homogenate was kept at $4^{\circ} \mathrm{C}$ for 1 hour, after which $50 \mu$ of serial dilutions were plated on tripticase soy agar (TSA). Bacterial count per organ was determined after 24 hours of incubation at $37^{\circ} \mathrm{C}$.

\subsection{Simultaneous Vancomycin Treatment}

In the simultaneous treatment study, the rats were weighed and anesthetized intraperitoneally (IP) with Ketamine $(100 \mathrm{mg} / \mathrm{kg}$ ) and xylazine $(10 \mathrm{mg} / \mathrm{kg})$ after which tail vein blood samples $(400 \mu \mathrm{l})$ were obtained. The rats were injected, IP, with $0.5 \mathrm{ml}$ of $S$. aureus $\left(1.0 \times 10^{8} \mathrm{cfu} / \mathrm{ml}\right)$. Immediately after bacterial injection, the rats were given subcutaneous (around the neck area) injections of drug treatment or control blank microspheres. Treatment was continued, IP, twice daily for three days thereafter. Blood samples were obtained at 4, 24, 48, 72, $96 \mathrm{hr}$ post infection, serially diluted, and plated overnight at $37^{\circ} \mathrm{C}$. On day five, the rats were euthanized, their organs removed and homogenates plated on agar.

\subsection{Delayed Vancomycin Treatment}

In a real case scenario, a patient is normally infected by a microorganism before treatment is initiated. The delayed treatment of infected rats mimics a real case situation. In this study, the rats were injected, IP, with $0.5 \mathrm{ml}$ of $S$. aureus $\left(1.0 \times 10^{8} \mathrm{cfu} / \mathrm{ml}\right)$. Four hours after infection, the rats were anesthetized, and tail vein blood samples were obtained. These were serially diluted and plated on agar. The rats were then given IP injections of drug microspheres, solution, or control. Tail vein blood samples were obtained at 8, 24, 48, 72, and $96 \mathrm{hr}$ post infection, serially diluted, and plated overnight at $37^{\circ} \mathrm{C}$. All the rats received their respective treatments twice a day for three days. On day five, the rats were euthanized, their organs removed and homogenates plated on agar.

\subsection{Statistical Analysis}

All data were analyzed using a two-tailed $t$-test.

\section{Results and Discussions}

\subsection{Exocytosis of Intracellular S. aureus from HMEC's}

The number of internalized bacteria increased steadily with time, however no bacteria was exocytosed in the first 5 hours of incubation (Figure 1). There was no analysis of the extracellular media between 5 and 24 hours, however after 24 hours of incubation, exocytosed bacteria was detected in the media. The number of exocytosed bacteria increased thereafter with time. These results were used to determine the incubation time of infected HMEC's prior to treatment with cytochalasin D. The number of intracellular $S$. aureus increased with time in the cytochalasin D untreated cells, whereas the number of intracellular bacteria decreased in the treated cells.

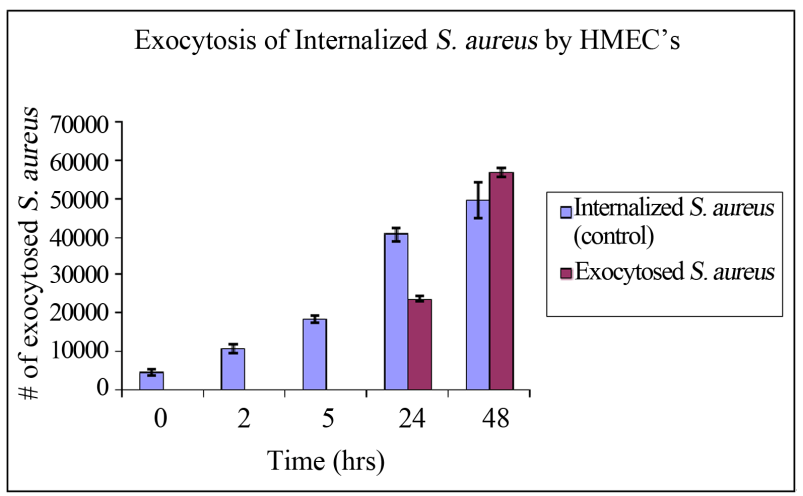

Figure 1. Internalized bacteria were eventually exocytosed from HMEC's. Exocytosis of internalized $S$. aureus occurs after 5 hours of incubation. Both the number of internalized and exocytosed bacteria increased with time. 
There was a significantly higher number of intracellular bacteria in the cytochalasin D untreated cells at 9 and 24 hours of incubation than in treated cells, $p<0.05$. The number of exocytosed bacteria from cytochalasin D untreated cells was significantly higher than those released from cytochalasin D treated cells at 7 and 24 hours of incubation (Figure 2). These results indicate that cytochalasin D was effective in inhibiting both uptake and exocytosis of $S$. aureus, hence actin polymerization is a possible mechanism of exocytosis. Our results confirm what others have found: That the actin cytoskeleton is actively involved in exocytosis [13].

\subsection{Effect of $S$. aureus and Its Cell Wall Products on HMEC permeability}

The effect of Lipoteichoic acid (LTA), peptidoglycan (Pep G), and S. aureus on the permeability of various molecular weight compounds through an endothelial cell monolayer was evaluated. Previous studies have shown that LTA can cause moderate hypotension in rats, but is unable to cause multiple organ failure or death by itself. [14] However, both LTA and pepG act in synergy to release TNF- $\alpha$ and gamma-interferon (IFN- $\gamma$ ), and to cause shock and multiple organ failure in anesthetized rats [15]. The first compound analyzed was FITC. PepG, PepG + LTA, and $S$. aureus were all significantly more effective than the control ( $1 \%$ HBSS) in increasing the permeation of FITC, $p<0.05$ (Figure 3). The permeation of FITCDextran through the HMEC monolayer showed a similar result as that seen in the case of FITC. FITC-Dextran,

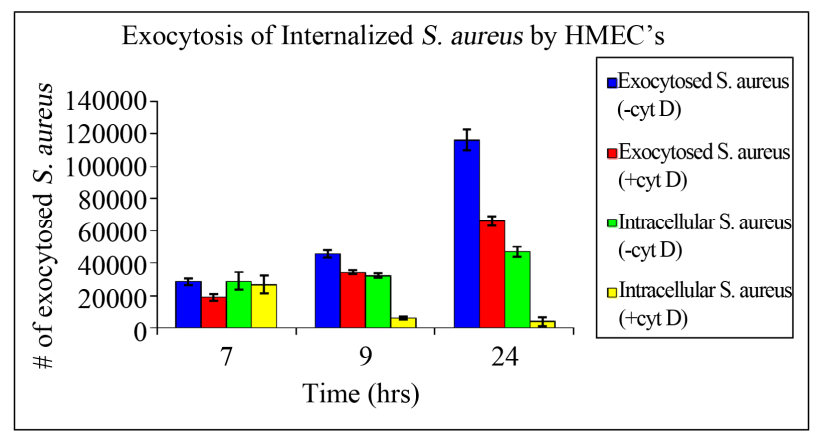

Figure 2. S. aureus infected HMEC's were divided into two groups. One group was treated with cytochalasin D $(1 \mu \mathrm{g} / \mathrm{ml})$ for 2 hours. Cytochalasin $D$ was washed off and the cells were incubated at $37^{\circ} \mathrm{C}$ and at various time points serial dilutions of the extracellular media were plated to determine number of exocytosed bacteria. Number of internalized bacteria was also determined. There was a significantly higher number of internalized bacteria by cells not treated with cytochalasin D ( - cyt D) than treated cells at 9 and 24 hours $\mathrm{p}<0.05$. The number of exocytosed $S$. aureus from HMEC's was significantly higher from cytochalasin D untreated cells than from the treated cells at 7 and 24 hours of incubation $\mathrm{p}<0.05$.

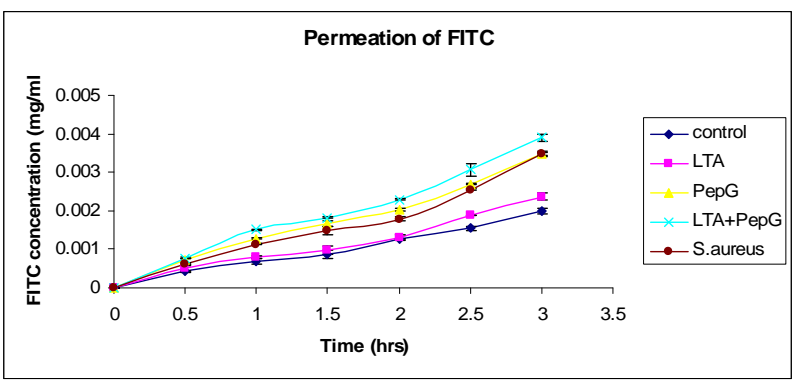

Figure 3. The effect of lipoteichoic acid (LTA), Peptidoglycan (PepG), and S. aureus (SA) on the permeation of FITC through HMEC monolayer. All the compounds used were more effective than the control (1\% HBSS) in increasing the permeation of FITC, however, only PepG, PepG + LTA, and $S$. aureus produced significantly higher effect than the control, $\mathrm{p}<0.05$.

being a higher molecular weight molecule than FITC alone, showed a rather slower rate and smaller amount of permeation through the monolayer. All the compounds used were more effective than the control (1\% HBSS) in increasing the permeation of FITC-Dextran, however, there was a significantly higher permeation effect only after 1 hour of incubation, $p<0.05$ (Figure 4). In the case of FITC-HSA permeation, no permeation of HSA was observed until after one hour of exposure, and this effect was observed after exposure to the combination of PepG + LTA. The combination of PepG + LTA was most effective in altering the monolayer integrity after three hours of exposure (Table 1). Table 2 shows the effect of lipoteichoic acid (LTA), Peptidoglycan (PepG), LTA + PepG, and S. aureus (SA) on the permeation of S. aureus through HMEC monolayer. Only LTA and LTA + PepG were effective in significantly increasing the permeation of $S$. aureus at all time points $\mathrm{p}<0.05$.

\subsection{Prophylactic Vancomycin Treatment}

Drug administration to a person prior to exposure to disease is rare, unless where vaccines are involved or when one expects to be exposed to the disease-causing pathogen. In the case of bacterial infections, treatment prior to exposure is even rarer; however, for hospital workers (in the case of nosocomial infections), pre-surgical wounds, and soldiers in the battlefield, prophylactic treatment with antibiotics might be necessary. In this study, treatment of rats prior to exposure to $S$. aureus showed that the presence of drug decreased the circulating bacterial load considerably. Moreover, vancomycin in the microsphere formulation was more effective in reducing the overall blood bacterial load than the solution form or blank microspheres. Between 4 and $24 \mathrm{hr}$ after infection, there was a decrease in bacteremia in all groups. This could be due to cellular uptake of circulating bacteria. Increase 


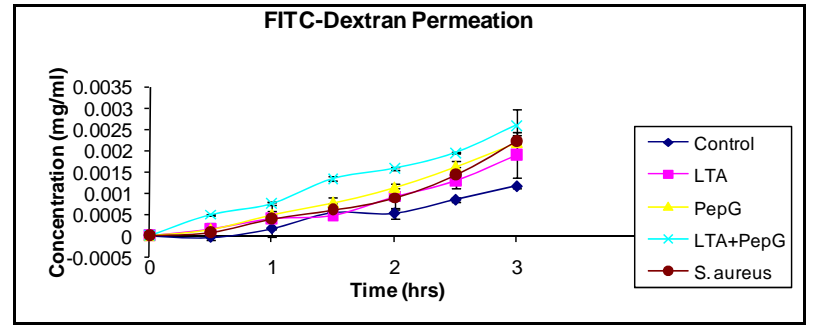

Figure 4. The effect of lipoteichoic acid (LTA), Peptidoglycan (PepG), and S. aureus (SA) on the permeation of FITCDextran through HMEC monolayer. All the compounds used were more effective than the control (1\% HBSS) in increasing the permeation of FITC-Dextran, however, there was a significantly higher permeation effect only after 1 hour of incubation, $\mathrm{p}<\mathbf{0 . 0 5}$.

Table 1. Effect of LTA, PepG, LTA + PepG and S. aureus on FITC-HSA permeation through endothelial monolayer.

\begin{tabular}{|c|c|c|c|c|c|}
\hline \multirow[b]{2}{*}{$\begin{array}{c}\text { Time } \\
(\mathrm{hr})\end{array}$} & \multicolumn{5}{|c|}{$\begin{array}{c}\text { FITC-HSA Concentration }\left(\times 10^{-3} \mathrm{mg} / \mathrm{ml}\right) \\
\text { Values are means } \pm \mathrm{SD}(\mathrm{n}=3)\end{array}$} \\
\hline & Control & LTA & Pep G & $\mathrm{LTA}+\mathrm{PepG}$ & SA \\
\hline 0 & $0.00 \pm 0.00$ & $0.00 \pm 0.00$ & $0.00 \pm 0.00$ & $0.00 \pm 0.00$ & $0.00 \pm 0.00$ \\
\hline 0.5 & $0.00 \pm 0.00$ & $0.00 \pm 0.00$ & $0.00 \pm 0.00$ & $0.00 \pm 0.00$ & $0.00 \pm 0.00$ \\
\hline 1.0 & $0.00 \pm 0.00$ & $0.00 \pm 0.00$ & $0.00 \pm 0.00$ & $0.21 \pm 0.30$ & $0.00 \pm 0.00$ \\
\hline 1.5 & $0.00 \pm 0.00$ & $0.00 \pm 0.00$ & $0.20 \pm 0.10$ & $2.00 \pm 0.40$ & $0.00 \pm 0.00$ \\
\hline 2.0 & $0.00 \pm 0.00$ & $0.00 \pm 0.00$ & ${ }^{*} 1.14 \pm 0.40$ & ${ }^{*} 2.07 \pm 0.40$ & $0.00 \pm 0.00$ \\
\hline 2.5 & $0.50 \pm 0.37$ & $0.09 \pm 0.10$ & *2.20 \pm 0.13 & *2.87 \pm 0.61 & $0.74 \pm 0.70$ \\
\hline 3.0 & $0.95 \pm 0.37$ & $1.50 \pm 0.99$ & ${ }^{*} 3.17 \pm 0.51$ & ${ }^{*} 3.96 \pm 0.41$ & $1.56 \pm 1.20$ \\
\hline
\end{tabular}

*Statistically significant difference at $\mathrm{p}<0.05$ compared with control (media).

Table 2. Effect of LTA, PepG, LTA+PepG and S. aureus on $S$. aureus permeation through endothelial monolayer.

\begin{tabular}{cccccc}
\hline & \multicolumn{5}{c}{ S. aureus colony forming units $/ \mathrm{ml}\left(\times 10^{-3} \mathrm{cfu} / \mathrm{ml}\right)$} \\
\cline { 2 - 6 } $\begin{array}{c}\text { Time } \\
\text { (hr) }\end{array}$ & Control & LTA & Pep G & LTA + PepG & SA \\
\hline 0 & $0.00 \pm 0.00$ & $0.00 \pm 0.00$ & $0.00 \pm 0.00$ & $0.00 \pm 0.00$ & $0.00 \pm 0.00$ \\
1.0 & $165 \pm 35$ & ${ }^{*} 225 \pm 22$ & $200 \pm 29$ & ${ }^{*} 314 \pm 6$ & $144 \pm 51$ \\
2.0 & $199 \pm 27$ & ${ }^{*} 299 \pm 13$ & $230 \pm 43$ & ${ }^{*} 396 \pm 16$ & $197 \pm 68$ \\
3.0 & $205 \pm 22$ & ${ }^{*} 352 \pm 30$ & $257 \pm 49$ & ${ }^{*} 454 \pm 4$ & $232 \pm 60$ \\
\hline
\end{tabular}

*Statistically significant difference at $\mathrm{p}<0.05$ compared with control (media).

in bacterial load after that could be due to exocytosis of internalized bacteria (Figure 5). Tissue analysis after five days of infection also confirmed the bacteremia results: Vancomycin microspheres were significantly more effective in reducing tissue bacteria load than either solution or blank microspheres (Figure 6). In all cases the bacterial load in the whole of the liver was higher than in either the spleen or the lung, however, the number of bacteria per gram of liver was less than in either organ.

\subsection{Simultaneous Vancomycin Treatment}

Simultaneous treatment of $S$. aureus infected rats produced similar results as the prophylactic treatment, although not as significant a difference. Vancomycin microspheres reduced the bacteremia levels more than either blank microspheres or vancomycin solution (Figure 7); however a significant difference in bacterial levels was seen only at 48 and 96 hours post infection. The presence of bacteria in the systemic circulation despite treatment could be due to either low systemic drug concentration or continued exocytosis of intracellular bacteria. It is possible that bacterial multiplication exceeded drug action.

Tissue analysis after five days of infection also showed that vancomycin microspheres were more effective in reducing intracellular bacterial load than either van-

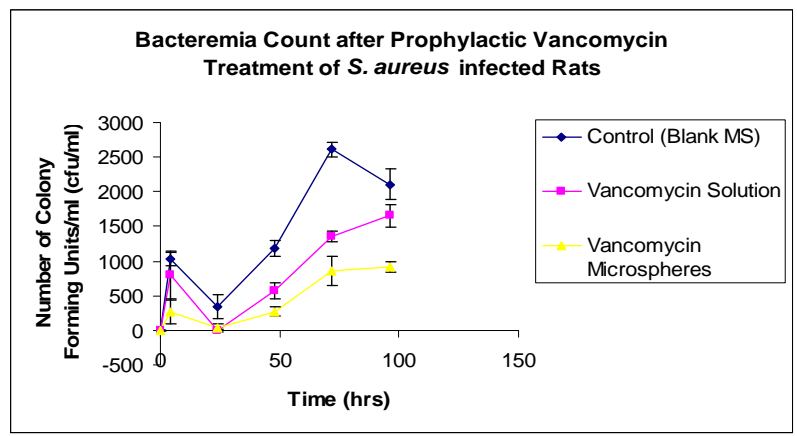

Figure 5. Effect of vancomycin microspheres and solution on circulating $S$. aureus. Treatment was initiated prior to bacterial infection. The microsphere formulation was more effective in reducing bacterial load than the solution form or control after 24 hours of infection. $p<0.05$.

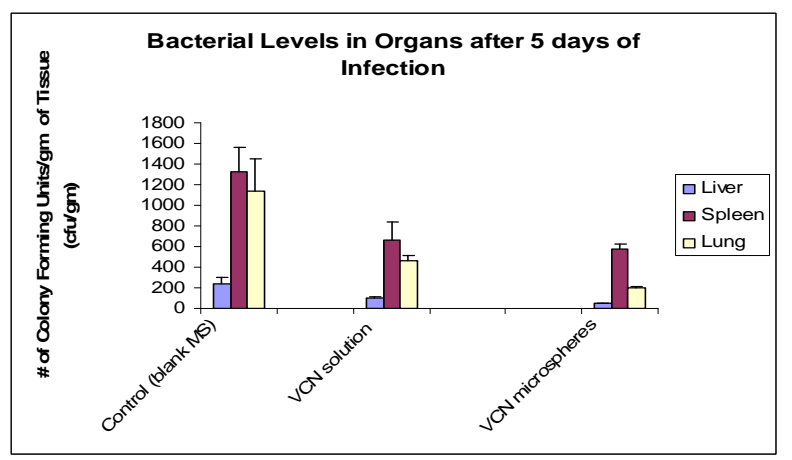

Figure 6. Bacterial levels in the liver, spleen and lung after 5 days of infection. Rats were treated with drug microspheres, solution or blank media for 4 hours prior to bacterial infection. 
comycin solution or blank microspheres (Figure 8). In all cases the blank microsphere group had the largest number of bacteria in the tissue.

\subsection{Delayed Vancomycin Treatment}

In the delayed treatment of $S$. aureus infected rats it was observed that the solution form was better than the microsphere form in reducing the plasma bacterial load after 8 hours of infection. It is expected that by the time treatment is initiated, the systemic and intracellular bacterial load would be very high due to bacterial multiplication. The dose of drug used was unchanged; hence the bacteremia count was high due to insufficient drug action. A possible explanation for the higher efficacy of vancomycin solution after eight hours of infection is that, the drug was readily available in the solution form. Drug release from the microsphere formulation increased with time thereafter and that was the reason for the decreased bacterial load at later time points. Vancomycin microspheres reduced the bacteremia load significantly more than either the solution or the blank microspheres at 48

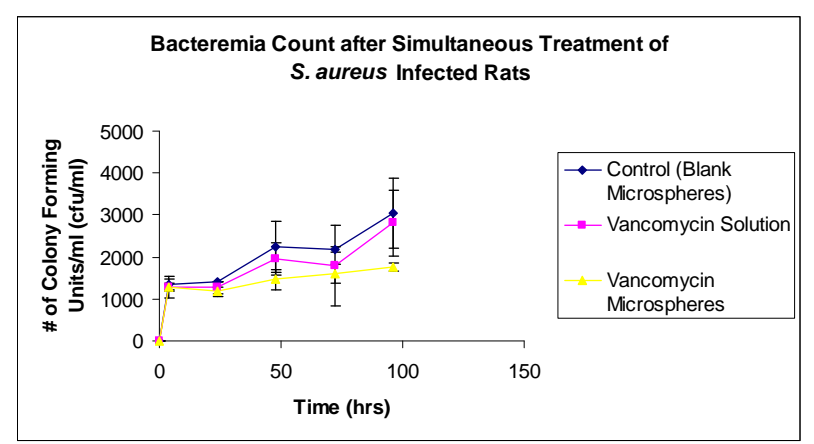

Figure 7. Effect of vancomycin microspheres and solution on circulating $S$. aureus. Treatment was initiated immediately after bacterial infection. The difference in effects was not significant $(p>0.05)$.

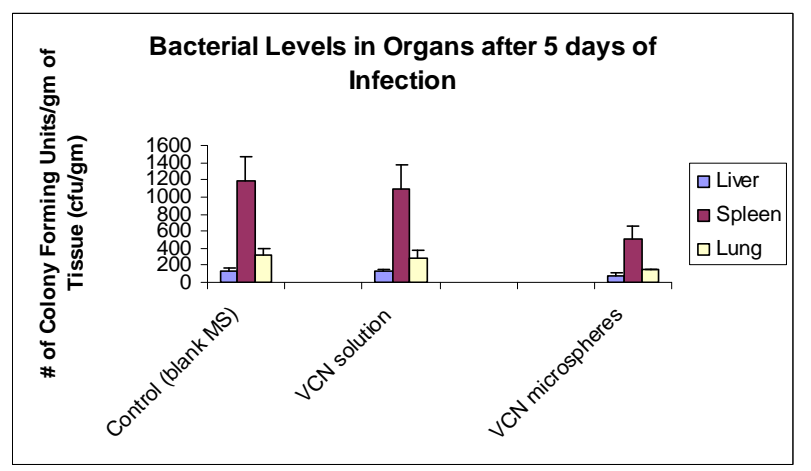

Figure 8. Bacterial levels in the liver, spleen and lung after 5 days of infection. Rats were treated with drug microspheres, solution or blank media immediately after bacterial infection. and 72 hours post infection (Figure 9).

Analysis of tissue samples excised after five days of infection showed that vancomycin microsperes were more effective than either solution or blank microspheres in reducing the bacterial load in the liver and spleen (Figure 10). There was no significant difference between the formulations in reducing the bacterial load in the lungs ( $p$ $>0.05)$.

\section{Conclusions}

It can be concluded from these results that $S$. aureus is exocytosed after it is taken up by endothelial cells. Exocytosis and phagocytosis of bacteria occur simultaneously, however, initially, the rate of phagocytosis far exceeds exocytosis. With increasing intracellular bacterial load, the exocytosis rate as well as extracellular bacterial multiplication exceeds phagocytosis. The partial inhibittion of exocytosis by cytochalasin D and the decreasing intracellular bacterial load shows that actin po-

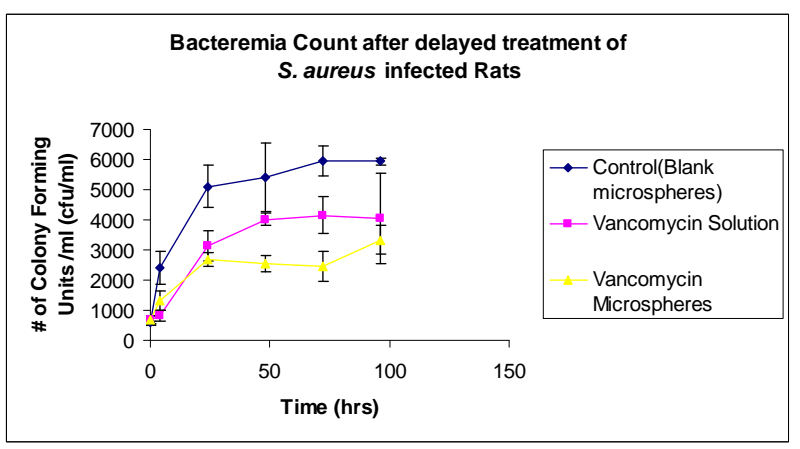

Figure 9. Effect of vancomycin microspheres and solution on circulating $S$. aureus. Treatment was initiated 4 hours after bacterial infection. Vancomycin microspheres were significantly more effective in reducing systemic bacterial load than the solution form or control at 48 and 72 hours post infection $(p<0.05)$.

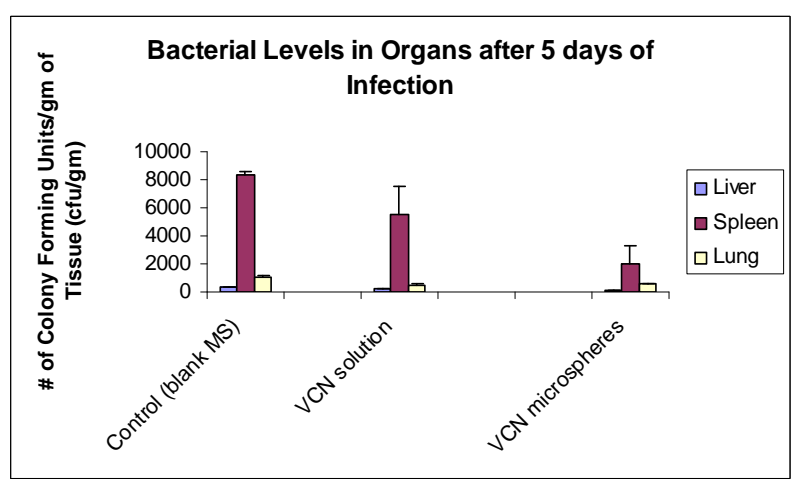

Figure 10. Bacterial levels in the liver, spleen and lung after 5 days of infection. Rats were treated with drug microspheres, solution or blank media 4 hours after bacterial infection. 
lymerization is a possible mechanism of exocytosis as well as phagocytosis. The profound effect that the combination of LTA and PepG had on the endothelial cell monolayer integrity shows that both components of the gram positive cell wall are required for vascular leakage to occur considerably.

In vivo challenge studies of peritonitis rat models supported previous in vitro results that vancomycin microspheres were more effective in killing intracellular bacteria [10]. In all three scenarios, namely prophylactic, simul-taneous, and delayed treatment, the microencapsulated form of vancomycin was more effective in reducing the systemic bacterial load than either blank microspheres or free vancomycin. Similar results were seen in the tissue analysis of bacteria levels.

The comparable distribution of both microspheres and bacteria in the spleen and liver will ensure effective killing of microbes (by encapsulated drugs) which infect these organs. In cases where the lungs are the infected organs, microspheres of larger sizes $(>7 \mu \mathrm{m})$ would be more effective. In sepsis, bacteria within endothelial cells are the main target; hence treatment would be more effective if professional phagocytes are initially saturated with blank microspheres, before treatment is initiated.

\section{Acknowledgements}

This study was supported by the Dialysis Clinic, Atlanta and Mercer University Southern School of Pharmacy.

\section{REFERENCES}

[1] R. G. Holzheimer, "The Significance of Endotoxin Release in Experimental and Clinical Sepsis in Surgical Patients - Evidence for Antibiotic-Induced Endotoxin Release?" Infection, Vol. 26, No. 2, 1998, pp. 77-84. doi:10.1007/BF02767765

[2] E. C. Hack, L. A. Aarden and L. G. Thijs, "Role of CytoKines in Sepsis," Advances in Immunology, Vol. 66, 1997, pp. 101-195. doi:10.1016/S0065-2776(08)60597-0

[3] R. J. Hamill, J. M. Vann and R. A. Proctor, "Phagocytosis of Staphylococcus aureus by Cultured Aortic Endothelial Cells: Model for Post-Adherence Events in Endovascular Infections," Infection and Immunity, Vol. 54, 1986, pp. 833-836.

[4] H. Beekhuizen, J. S. Van de Gevel, B. Olsson, I. J. Van Benten and R. Van Furth, "Infection of Human Vascular Endothelial Cells with Staphylococcus aureus Induces Hyperadhesiveness for Human Monocytes and Granulocytes," The Journal of Immunology, Vol. 158, No. 2, 1997, pp. 774-782.

[5] E. C. Hack and M. D. Zeerleder, "The Endothelium in Sepsis: Source of and a Target for Inflammation," Critical Care Medicine, Vol. 29, No. 7, 2001, pp. S21-S27. doi:10.1097/00003246-200107001-00011
[6] M. D’Souza, C. W. Oettinger and G. V. Milton, "Microspheres Containing Neutralizing Antibodies to Tumor Necrosis Factor- $\alpha$ and Interleukin- $1 \beta$ Protect Rats from Staphylococcus aureus-Induced Peritonitis," Journal of Interferon \& Cytokine Research, Vol. 20, No. 10, 2000, pp. 907-913. doi:10.1089/10799900050163280

[7] P. K. Gupta, C. T. Hung and D. G. Perrier, "Albumin Microspheres I: Release Characteristics of Adriamycin," International Journal of Pharmaceutics, Vol. 33, No. 1-3, 1986, pp. 137-146. doi:10.1016/0378-5173(86)90047-5

[8] M. D'Souza and P. DeSouza, "Preparation and Testing of Cyclosporine Microsphere and Solution Formulations in the Treatment of Polyarthritis in Rats," Drug Developments in Industrial Pharmacy, Vol. 24, No. 9, 1998, pp. 841-852. doi:10.3109/03639049809088529

[9] M. D'Souza and R. Pourfazib, "Improved Efficacy of a Microencapsulated Macrophage Colony-Stimulating Factor and Methotrexate in Melanoma," Drug Developments in Industrial Pharmacy, Vol. 25, No. 5, 1999, pp. 583590. doi:10.1081/DDC-100102212

[10] H. Nettey, D. Haswani, C. Oettinger and M. J. D’Souza, "In Vitro Antimicrobial Effect of Encapsulated Vancomycin on Internalized Staphylococcus aureus within Endothelial Cells," Drug Development and Industrial Pharmacy, Vol. 33, No. 2, 2007, pp. 133-139. doi: $10.1080 / 03639040600867989$

[11] V. Schafer, H. Briesen, H. Rubsamen-Waigman, A. M. Steffan, C. Royer and J. Kreuter, "Phagocytosis and Degradation of Human Serum Albumin Microspheres and Nanoparticles in Human Macrophages," Journal of Microencapsulation, Vol. 11, No. 3, 1994, pp. 261-269. doi:10.3109/02652049409040455

[12] H. Nettey, D. Haswani, C. Oettinger and M. J. D'Souza, "Formulation and Testing of Vancomycin Loaded Albumin Microspheres Prepared by Spray-Drying," Journal of Microencapsulation, Vol. 23, No. 6, 2006, pp. 632-642. doi:10.1080/02652040600776564

[13] S. Gasman, S. Chasserot-Golaz, N. Vitale and M. F. Bader, "Bacterial Toxins: Useful for Studying G-Proteins Implicated in the Mechanism of Exocytosis in Neuroendocrine Cells," Journal de la Société de Biologie, Vol. 193, No. 6, 1999, pp. 451-456

[14] S. J. De Kimpe, M. L. Hunter and C. F. Bryant, "Delayed Circulatory Failure Due to the Induction of Nitric Oxide Synthase by Lipoteichoic Acid from Staphylococcus aureus in Anaesthetized Rats," British Journal of Pharmacology, Vol. 114, No. 6, 1995, pp. 1317-1323. doi:10.1111/j.1476-5381.1995.tb13349.x

[15] S. J. De Kimpe, M. Kengatharan, C. Thimermann and J. R. Vane, "The Cell Wall Components Peptidoglycan and Lipoteichoic Acid from Staphylococcus aureus Act in Synergy to Cause Shock and Multiple Organ Failure," Proceedings of the National Academy of Sciences, Vol. 92, No. 22, 1995, pp. 10359-10363. doi:10.1073/pnas.92.22.10359 